\title{
SMALL ADENOCARCINOMA OF THE CERVIX WITH SINGLE GIANT OVARIAN METASTASIS: CASE REPORT
}

Micelli, L.P.; Nobrega, F.S.; Carvalho, J.P.M.; Genta, M.L.N.D.; Piato, D.S.A.M.; Suárez, G.M., Carvalho, J.P.

Instituto do Câncer do Estado de São Paulo (ICESP)

\section{OBJECTIVES:}

There were few studies regarding the incidence of ovarian metastasis of adenocarcinoma of the cervix. The data show occurrence varying between 0 and $12,6 \%$ with no explicit risks factors ${ }^{1}$. This cases have the objective to demonstrate the importance of ovarian evaluation in cases of adenocarcinoma of the cervix, since the ovaries may be the only site of metastasis.

\section{METHODS:}

Analysis of patient medical record.

\section{CASE REPORT:}

A 56-year-old female presented to the outpatient clinic with increased abdominal volume.

Gynecological examination revealed a small exophytic lesion of $2 \mathrm{~cm}$ in the endocervical canal, without vaginal or parametrial involvement. Biopsy of the lesion resulted in adenocarcinoma with papillary and glandular components and immunohistochemistry proving primary tumor of the uterine cervix.

The patient underwent a pelvic mangnetic resonance (Fig. 1-3) and computed tomography (Fig. 4-6). Bilateral ovarian masses with solid components and ascites with no signs of carcinomatosis were observed.

The patient was submitted to radical hysterectomy with bilateral salpingoophorectomy, pelvic lymphadenectomy, omentectomy, appendectomy and multiple peritoneal biopsies.

Anatomopathological analysis resulted in usual endocervical adenocarcinoma with intestinal pattern in the cervix and cervical metastasis in both ovaries. All surgical margins were free of disease.

\section{CONCLUSIONS}

This case was an unusual presentation of cervical cancer that showed the possibility of single ovarian metastasis without any other tumor involvement. The importance of adequate preoperative ovarian evaluation is highlighted, raising the question whether there is a role for bilateral oophorectomy in all cases of adenocarcinoma of the cervix.

1- Hu J1, Jiao X1, Yang Z1, Cui H2, Guo H3, Wu Y4, Zhu L1. Should ovaries be removed or not in early-stage cervical adenocarcinoma: a multicenter retrospective study of 105 patients. J Obstet Gynaecol. 2017; 37(8):1065-1069.
Fig. 1 and 2 - Cervical tumor (restrict to the cervix)
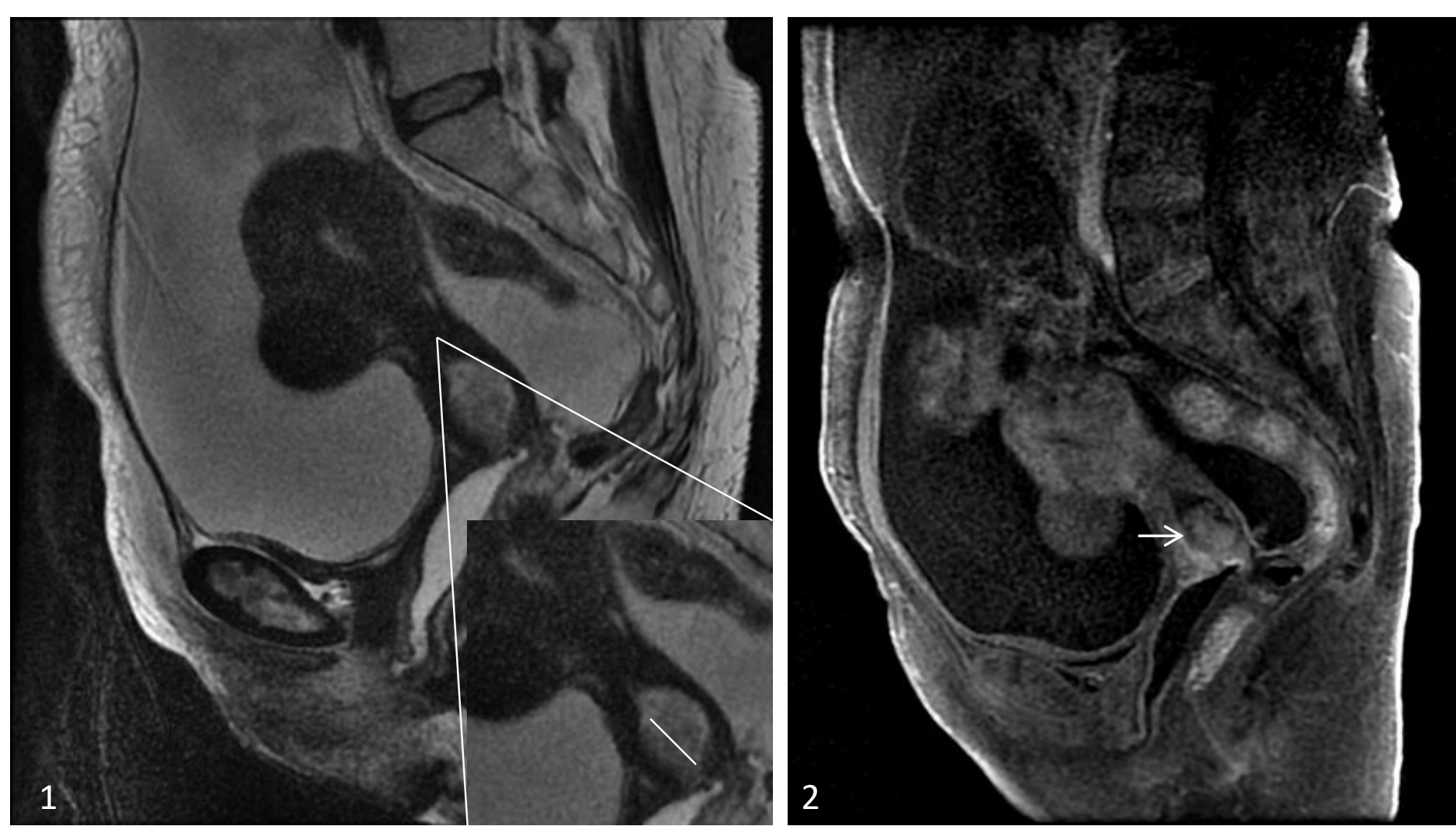

Fig. 3 - Cervical tumor (without parametrial invasion)

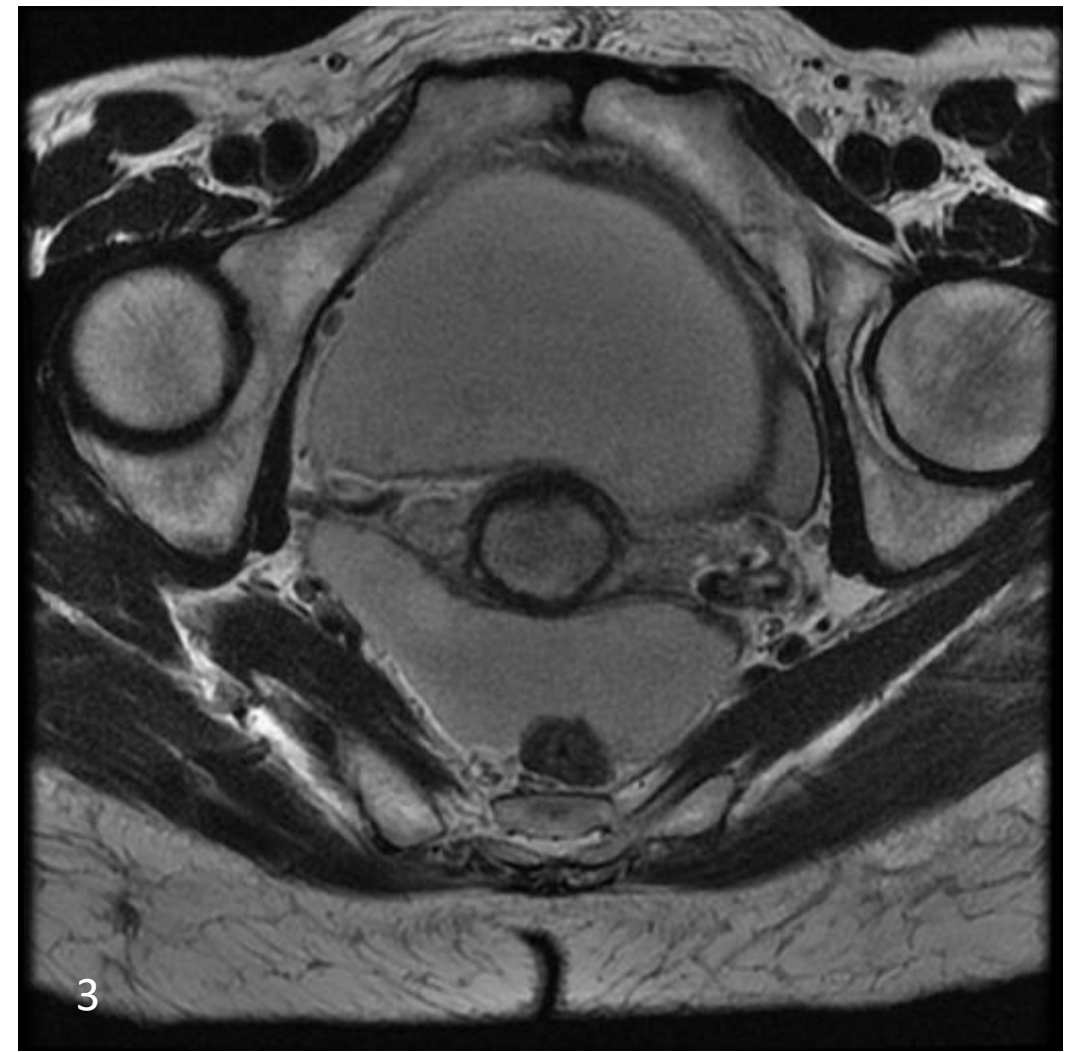

Fig. 4 - Bilateral ovarian masses (arrow) and cervical tumor (line)

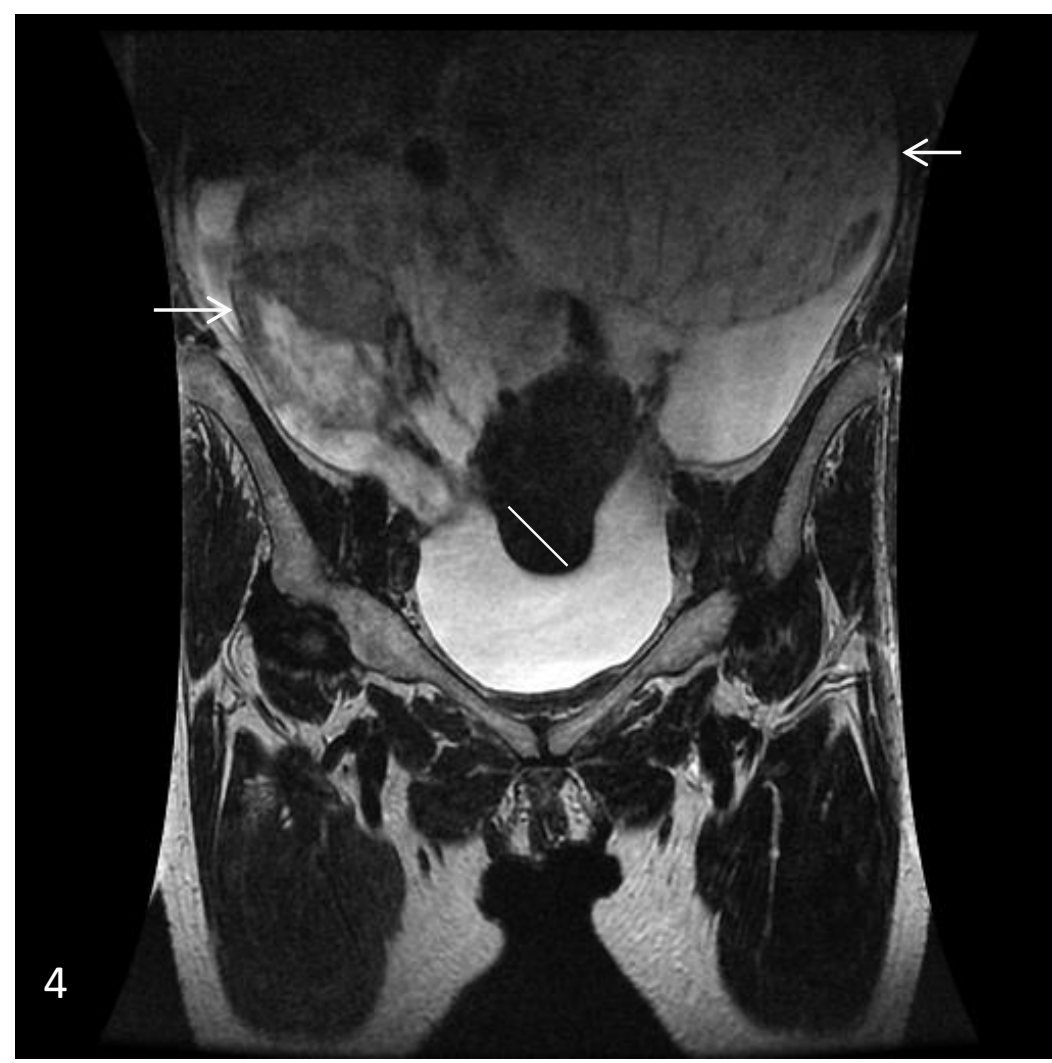

Fig. 5 and 6 - Bilateral ovarian masses (arrow)
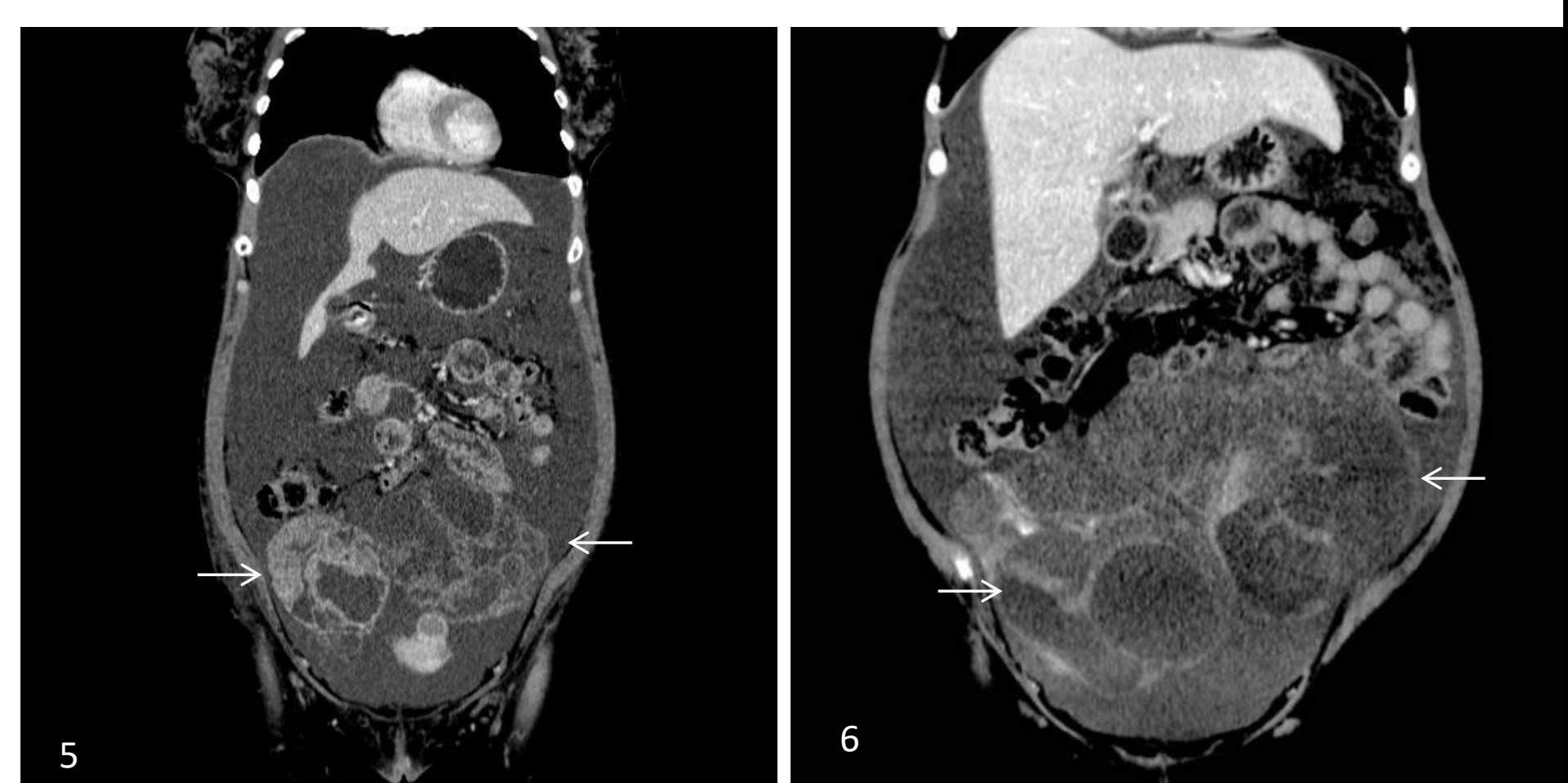